\title{
Anticoagulation and Vessel Recanalization in Cirrhotic Patients with Splanchnic Vein Thrombosis: A Multidisciplinary "Real Life" Experience
}

\author{
Serena Rupoli ${ }^{1}$ \\ Alessandro Fiorentini' \\ Erika Morsia' \\ Gianluca Svegliati-Baroni ${ }^{2}$ \\ Giorgia Micucci' \\ Luca Maroni ${ }^{2}$ \\ Kimberly Blaine Garvey' \\ Alessandro Fiorentini Snr ${ }^{3}$ \\ Alessandra Riva Snr ${ }^{4}$ \\ Lidia Da Lio ${ }^{5}$ \\ Antonio Benedetti ${ }^{2}$ \\ Massimo Offidani (D) \\ Attilio Olivieri $^{1}$ \\ Tarantino Giuseppe ${ }^{2}$ \\ 'Clinica Ematologica, Dipartimento di \\ Medicina Interna, Ancona, Italy; ${ }^{2} \mathrm{SOD}$ \\ Clinica di Gastroenterologia, Epatologia \\ ed Endoscopia Digestiva d'Urgenza, \\ Ancona, Italy; ${ }^{3}$ SOD Malattie Infettive \\ emergenti e degli immunodepressi, \\ Ospedali Riuniti Ancona, Ancona, Italy; \\ ${ }^{4}$ SOD Clinica Malattie Infettive tropicali, \\ parassitologia, epatiti croniche, Ancona, \\ Italy; ${ }^{5}$ SOD Medicina di Laboratorio, \\ Ospedali Riuniti Ancona, Ancona, Italy
}

Correspondence: Erika Morsia Clinica Ematologica, Ospedali Riuniti Ancona, via Conca 7I, 60I26, Italy Email erika.morsia@ospedaliriuniti. marche.it
Background and Aim: Splanchnic vein thrombosis (SVT) is a potentially life-threatening complication of liver cirrhosis. This study aimed to evaluate the impact of a multidisciplinary approach and early anticoagulation therapy (AT) on bleeding/thrombotic events, recanalization rates and outcome of cirrhotic patients with SVT.

Methods: This is a single-center, registry-based cohort study. Over 17 years, 149 SVT patients were enrolled and prospectively evaluated. Regarding cirrhotic-SVT, a pre-specified algorithm, guiding initial posology of AT and follow-up visits schedule, was performed. Major bleeding (MB), thrombotic events, functional liver scores and all cause-mortality were investigated. Efficacy of AT was evaluated by radiological imaging.

Results: In cirrhotic-SVT, the incidence rate of MB was 8.4 per 100 patient-year $(95 \% \mathrm{CI}$, 3.83-15.97), while the incidence rate of thrombosis was 5.6 per 100 patient-year $(95 \% \mathrm{CI}$, 2.05-12.2). In incidental SVT treated with AT, MB incidence was 6.5 per 100 patient-year (95\% CI: 2.8-12.82), while in symptomatic SVT was 2.2 per 100 patient-year (95\% CI: 0.25-8.02). All thrombotic recurrences occurred in incidental SVT (7.7 per 100 patientyears; 95\% CI, 3.71-14.26). Overall survival was significantly higher in patients who had at least a partial recanalization $(\mathrm{p}<0.01)$ and partial/total recanalization was independently associated with improved MELD score at multivariate analysis (HR 2.62, 95\% CI 1.1-6.47, $\mathrm{p}=0.03)$.

Conclusion: In cirrhotic SVT patients, partial or total resolution of thrombosis ameliorates liver function and is associated with higher overall survival. A multidisciplinary approach together with radiological follow-up at pre-fixed time improves patient selection and monitoring.

Keywords: splanchnic vein thrombosis, cirrhosis, anticoagulant therapy, vessel recanalization, multidisciplinary approach

\section{Introduction}

Splanchnic Vein Thrombosis (SVT) refers to a life-threatening type of Venous Thrombo-Embolism (VTE) including portal, mesenteric (superior and inferior), splenic vein thrombosis and the Budd-Chiari syndrome. ${ }^{1}$ In a real-life setting, the major risk factors for SVTs are liver cirrhosis and solid cancers, each responsible for about $25 \%$ of cases. ${ }^{2,3}$ A high variability in the incidence of SVT has been reported, in relation to the type of data source, differences between diagnostic studies, site or type of SVT. ${ }^{4}$ Patients may be asymptomatic or present different symptoms depending on the site and the extent of the obstruction, the rapidity of its 
development, and the presence of predisposing underlying conditions. In this setting, SVT is classified as provoked if secondary to a local or systemic risk factor, and unprovoked if the causative trigger is not found. ${ }^{5,6}$ The quality of evidence guiding the treatment of SVT is low, due to the lack of randomized trials and the large heterogeneity of published data (different patient populations, timing of AT, drug selection and dosages $)^{7-10}$. Moreover, in the realworld clinical practice, a proportion of SVT patients are left untreated because the risks associated with AT are felt by physicians to exceed the benefits guidelines of the American Association for the Study of Liver Diseases (AASLD), published in 2009, stated that, due to limited data on the benefit of the AT, the therapeutic decision should be made on a case-by-case basis. ${ }^{10}$ The guidelines of the American College of Chest Physicians (ACCP), published in 2012, formulated only general recommendation, without any distinction for cirrhotic patients. ACCP broadly recommended anticoagulation in patients with symptomatic SVT and no anticoagulation in incidentally detected SVT. ${ }^{9}$

The Baveno VI consensus workshop, published in 2015, recommended AT in cirrhotic patients with SVT only in those who are potential candidates for liver transplant. ${ }^{8}$ Regarding the optimal duration of secondary prevention of SVT, several guidelines suggest 3-6 months of AT in patients with SVT provoked by transient reversible risk factors, while a longer indefinite treatment duration is recommended for patients with permanent predisposing conditions, unprovoked thrombosis or particularly severe disease (such as biliary cast syndrome). ${ }^{6,11,12}$ While a definite goal of anticoagulation is vessel recanalization, it remains unclear whether recanalization of splanchnic veins could positively influence the course of chronic liver disease, decrease the risk of gastrointestinal bleedings and improve survival outcomes. Here, we describe a multi-disciplinary management in a cohort of 119 cirrhotic-associated SVT. Long-term clinical outcomes (MB episodes, thrombotic events, and mortality), recanalization rate at radiological follow-up and the impact of recanalization on liver dysfunction and survival are reported.

\section{Patients and Methods Study Design and Population}

This is a single-center, cohort analysis of data prospectively entered into the SVT registry of Ospedali Riuniti of Ancona (Marche, Italy), resulting from an interdisciplinary collaboration between Gastroenterology, Infectious Disease and Haematology departments.

The diagnosis of SVT was objectively confirmed by imaging tests (Doppler ultrasound, angiography, computed tomography or magnetic nuclear resonance) or documented during laparoscopic/abdominal surgery. The diagnosis of cirrhosis was established from medical history, liver radiology, and liver biopsy or non-invasive assessment of fibrosis, when available. We analyzed a cohort of 149 consecutive patients with SVT (119 patients with cirrhotic SVT; 30 patients with non-cirrhotic SVT), diagnosed from the time of the first enrollment in January 2000 to the last, in October 2017. Patients receiving prior thrombolysis/ thrombectomy were excluded from the study.

For cirrhosis-associated SVT, a pre-specified algorithm, guiding initial posology of low-molecular weight heparin (LMWH), vitamin $\mathrm{K}$ antagonists (VKA) or Fondaparinux and follow-up visit schedule was applied. Conversely, decisions regarding the type of anticoagulant drug, the duration of the treatment and the timing of radiological follow-up scans were left to the discretion of attending clinicians (Figures 1 and 2).

All patients included in the study were informed and provided consent for prospective data collection at the time of SVT diagnosis. The pre-specified study protocol conforms to the ethical guidelines of the 1975 Declaration of Helsinki and was approved by the local ethical committee (Ethics Committee of Azienda Ospedaliera Universitaria Ospedali Riuniti, Ancona). For liver transplant recipients, all organs were donated voluntarily with written informed consent, in accordance with the Declaration of Istanbul.

\section{Baseline Evaluation}

Before starting AT in patients with cirrhotic SVT, an esophagogastroduodenoscopy (EGD) was performed and F2/F3 esophageal varices were treated with appropriate prophylaxis (beta-blockers and/or endoscopic band ligations to the discretion of gastroenterologist).

In the absence of the most relevant bleeding risk factors, AT was started as soon as possible, in all cases of SVT (both complete and partial, symptomatic or incidental) regardless of the etiological diagnosis. More in details, anticoagulation was avoided for platelet count lower than $30,000 / \mu \mathrm{L}$, Glomerular Filtration Rate (GFR) $<30 \mathrm{~mL} / \mathrm{min}$ or active gastrointestinal bleeding. Conversely, for platelet count between 30 and $50,000 / \mu \mathrm{L}$ or GFR $30-50 \mathrm{~mL} / \mathrm{min}$, we used $50 \%$ of therapeutic dose or prophylactic dose of $\mathrm{LMWH} /$ fondaparinux. 


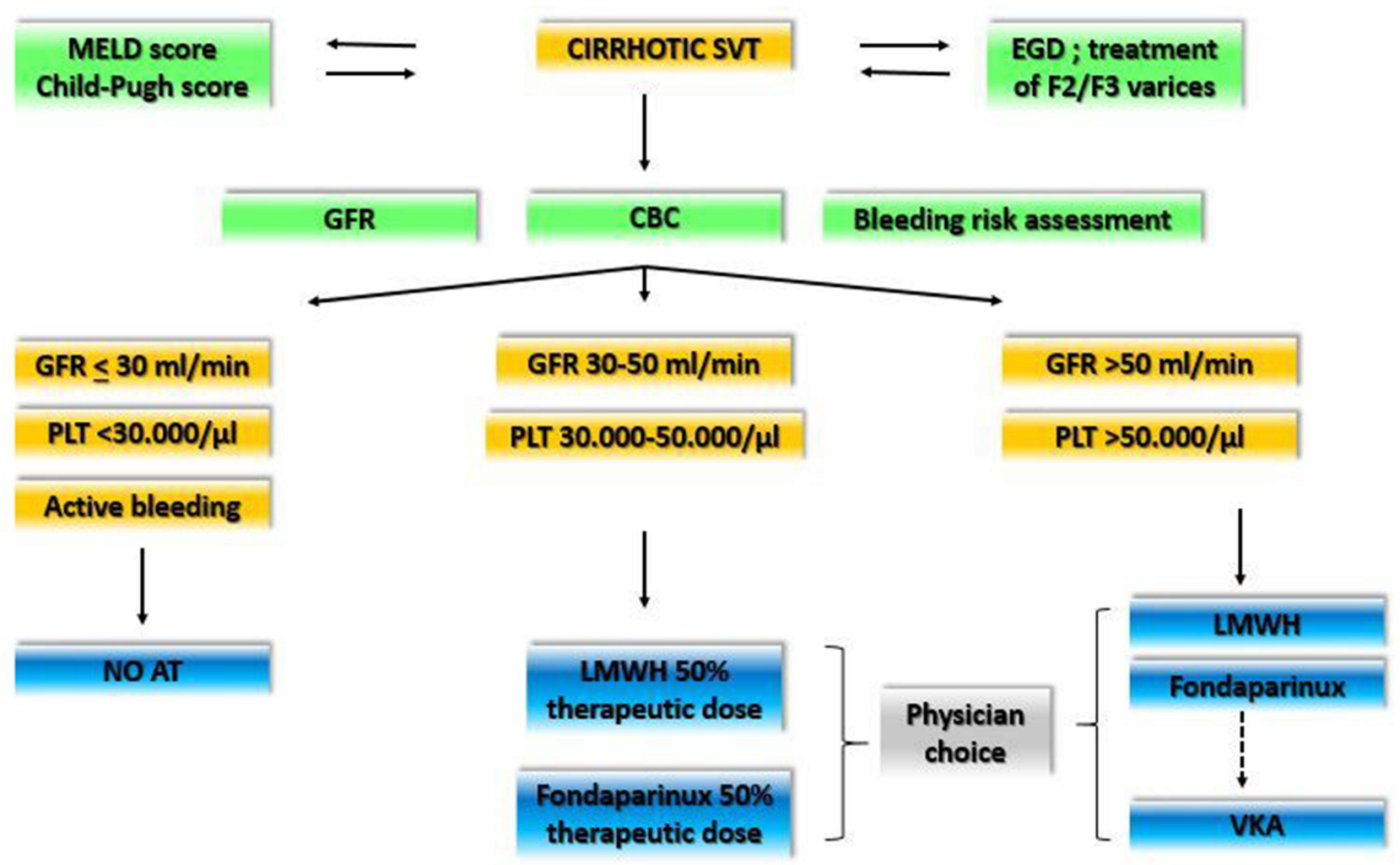

Figure I Baseline multi-disciplinary approach to SVT.

Abbreviations: AT, Anticoagulant treatment; CBC, Complete blood count; GFR, Glomerular filtration rate; EGD, Esophagogastroduodenoscopy; LMWH, Low Molecular Weight Heparin; VKA, Vitamin K Antagonists.

For platelet count $\geq 50,000 / \mu \mathrm{L}$ and GFR $>50 \mathrm{~mL} / \mathrm{min}$, we started anticoagulation with full dose of $\mathrm{LMWH} /$ fondaparinux with or without an early switch to a VKA, with a target INR 2.5 or lower (2.2, range 1.8-2.5) in patients with higher bleeding risk (Figure 1).

\section{Clinical Follow-Up}

The AT was monitored by our Haemostasis and Thrombosis center. A pre-planned visit schedule to periodically reassess bleeding risk and efficacy of AT was adopted (Figure 2). The initial treatment algorithm was followed at every pre-planned visit. The choice to repeat an EDG was left to the discretion of attending clinicians (eg, if an increased bleeding risk from baseline was noted).

\section{Radiological Follow-Up}

Both in AT and non-AT patients, the option to assess the status of the SVT, by CT scan, was left to attending physicians, both in AT and non-AT patients.

All CT images were retrospectively reviewed by local expert radiologists, aware of the diagnosis but blinded concerning the treatment the patient received, to establish the rate of recanalization in a univocal manner. The patency of the splanchnic venous system at follow-up CT was categorized as progression, stability, partial regression, and complete regression. If patients underwent multiple CT scans until complete recanalization, the time of last imaging test was considered as the time of recanalization.

\section{Procedures and Outcomes}

The following baseline data were collected from patients at the time of inclusion in the study: demographic characteristics, personal history of previous VTE, comorbidities, cardiovascular risk factors, a complete blood count, results of inherited and acquired thrombophilia tests, mutational profile for Myeloproliferative Neoplasms (BCRABL and JAK2 V617F mutation), imaging and endoscopic tests at baseline. Child-Pugh and MELD scores were used to assess the severity and prognosis of cirrhosis at diagnosis and at every follow-up scheduled visit and considered "improved" if at last follow-up visit they were lower than those calculated at baseline.

Information on type, dose, duration of anticoagulant AT, clinical outcomes (major bleeding (MB), thrombotic 


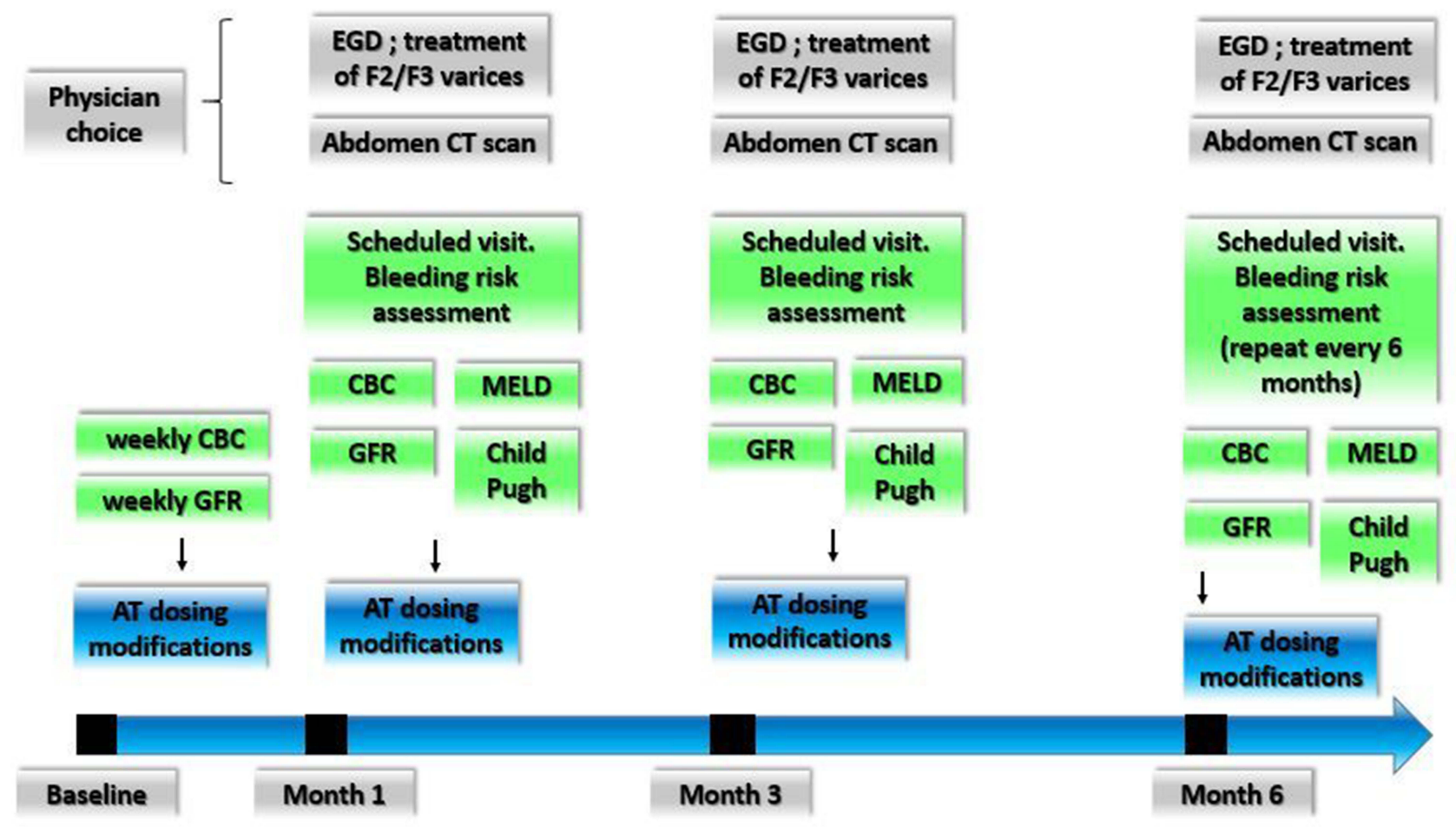

Figure 2 Follow-up schedule evaluation of SVT.

Abbreviations: AT, Anticoagulant treatment; CBC, Complete blood count; GFR, Glomerular filtration rate; EGD, Esophagogastroduodenoscopy.

recurrence, recanalization and mortality) was also periodically recorded (Figure 2).

MB was defined according to the criteria of the International Society on Thrombosis and Haemostasis: ${ }^{13}$ fatal and/or symptomatic haemorrhage in a critical area, reduction of $2 \mathrm{~g} / \mathrm{dl}$ or more in haemoglobin level or bleeding leading to transfusion of two or more blood units were regarded as MB; all other bleedings were considered as minor.

Recurrent thrombosis was recorded only if objectively documented. Recurrent SVT was defined as thrombus occurrence in a previously patent venous vessel, or venous thromboembolism at other sites or mesenteric infarction revealed on a pathology specimen, or any of the following arterial thrombosis (acute coronary syndromes, acute ischemic stroke, transient ischemic attack, and acute peripheral arterial disease) diagnosed according to standard criteria. If the patients presented to another health-care institute, outcome events (deaths, MB and thrombosis recurrence) were recorded after reviewing medical charts.

\section{Statistical Analysis}

Continuous variables were expressed as mean \pm standard deviation. Those with a non-parametric distribution were expressed as medians, indicating, where appropriate, the minimum and maximum values observed. Categorical variables were expressed as frequency and percentage of the cases. The differences were calculated using the "Student's t" and the "Mann-Whitney" tests for continuous variables and the "chi-square" test for categorical ones. The number of events during follow-up was expressed as incidence rate and calculated as the number of new cases per 100 patient-years (pt-y) with $95 \%$ confidence interval (CI), calculated with Byar-Poisson approximation. Moreover, we calculated the "on treatment" and "off-treatment" incidence of clinical events. The primary analysis was performed up to the first confirmed clinical outcome (major bleeding episode or thrombotic event).

To explore the role of potential predictors of vessel recanalization and mortality, different multivariable Cox's proportional hazards regression models were analyzed, using backwards stepwise removal of the variables $(\mathrm{P}<$ 0.05 for inclusion and $\mathrm{P}>0.10$ for exclusion, respectively). The initial variables considered for analysis were: presence of Hepatocellular Carcinoma (HCC) or solid cancer, ascites, esophageal varices, age $>60$ years old, incidental SVT. The same approach was used to evaluate 
potential predictors of Child-Pugh and MELD score improvement from baseline to last follow-up, with initial variables being: presence of HCC or solid cancer, ascites, esophageal varices, age $>60$ years old and improvement of SVT at first imaging test. The variables with a significance level of $\mathrm{P}<0.05$ at the multivariate analysis were considered to be associated with the outcome of interest. Overall survival rates and their association with radiological status of SVT were assessed with the Kaplan-Meier method. Statistical analysis was performed with SPSS software $26.0^{\circledR}$ and graphics and figures formulated with Excel and PowerPoint $2016^{\circledR}$, respectively.

\section{Results}

\section{Descriptive Analysis}

The whole population enrolled in the registry was composed by 149 patients with SVT (74\% males; median age of 60 years old). Cirrhotic SVT patients were 119 and median follow-up period for cirrhotic patients was 14.5 months (range 3-231 months). Details regarding the family history, risk factors, sites of thrombosis, and other baseline characteristics of these patients are reported in Table 1. In particular, 91 were males (76\%) and 28 females (24\%); the aetiologies of cirrhosis included: chronic HCV infection $(\mathrm{n}=38 ; 32 \%)$, alcohol-related liver disease $(\mathrm{n}=29$; $24 \%)$, non-alcoholic steatohepatitis $(n=35 ; 29 \%)$, chronic HBV infection $(n=9 ; 8 \%)$, primary biliary cholangitis $(\mathrm{n}=3 ; 2 \%), \mathrm{HBV} / \mathrm{HCV}$ coinfection $(\mathrm{n}=1 ; 1 \%)$, hemochromatosis $(n=1 ; 1 \%)$, secondary biliary cirrhosis $(n=2 ; 2 \%)$, Caroli disease $(\mathrm{n}=1 ; 1 \%)$. At the time of thrombosis, most patients were in Child-Pugh classes A and B (Child-Pugh A $n=36(30 \%)$; Child-Pugh B $n=59(50 \%)$; only 24 were in Child-Pugh (20\%); the median Child-Pugh score was 8 (range, 5-12) and the median MELD score was 12 (range, 6-38). Overall, 57 patients $(48 \%)$ had F2 or F3 grade esophageal varices. HCC was found in 41 patients (34.5\%). SVT was total/occlusive in 90 cirrhotic patients $(76 \%)$. The most common sites of thrombosis were the portal vein $(55 \%)$ followed by multiple sites $(34 \%)$. SVT was incidentally detected in 83 cirrhotic patients $(68 \%)$.

\section{Treatment}

Ninety-six of 119 patients (81\%) started anticoagulation, according to the established criteria; the most commonly used anticoagulant drug was LWMH (72\%) at intermediate-therapeutic or prophylactic doses $(58 \%$ and $42 \%$, respectively); VKA (usually after an initial course of
Table I Main Characteristics of 119 Cirrhotic Patients at Diagnosis of SVT. Data are Expressed as Median (Interquartile Range) or Number (Percentage)

\begin{tabular}{|l|l|}
\hline Age, years (median, range) & $60(18-90)$ \\
\hline Gender, male (n, \%) & $91(76 \%)$ \\
\hline Family history of VTE (n, \%) & $1(1 \%)$ \\
\hline Clinical presentation (n, \%) & \\
Symptomatic & $36(32 \%)$ \\
Incidentally Detected & $83(68 \%)$ \\
\hline Involved veins (n, \%) & \\
Portal VT & $66(55 \%)$ \\
Mesenteric VT & $8(7 \%)$ \\
Splenic VT & $1(1 \%)$ \\
Suprahepatic VT & $3(3 \%)$ \\
Multiple Veins & $41(34 \%)$ \\
JAK2 V6I7F or BCR/ABL + (n, \%) & $0(0 \%)$ \\
\hline Esophageal varices (n, \%) & \\
FI & $34(29 \%)$ \\
F2 & $44(37 \%)$ \\
F3 & $13(11 \%)$ \\
Solid Cancer (n, \%) & $48(40 \%)$ \\
Hepatocarcinoma (n, \%) & $41(35 \%)$ \\
Previous abdominal surgery (n, \%) & $10(8 \%)$ \\
Abdominal inflammation/infection (n, \%) & $5(4 \%)$ \\
Hormonal therapy (n, \%) & $3(3 \%)$ \\
Abnormal trombophilia test (n, \%) & $20(17 \%)$ \\
Cronic kidney disease (n, \%) & $24(20 \%)$ \\
Platelet Count at diagnosis, $\mathrm{n} \times 10 * 3$ (median, range) & $79(27-558)$ \\
\hline Child Pugh Score at diagnosis (n, \%) & $69(58 \%)$ \\
A & $34(12 \%)$ \\
B & $36(30 \%)$ \\
C & $59(50 \%)$ \\
\hline MELD score at diagnosis (n, \%) & $24(20 \%)$ \\
\hline I0 & \\
I0-I8 & \\
\hline I8 & \\
\hline
\end{tabular}

Abbreviations: IQR, interquartile range; SVT, splanchnic vein thrombosis; VTE, venous thromboembolism.

parenteral treatment) and fondaparinux were used in $22 \%$ and $6 \%$ of cases, respectively (Table 2). Twenty-three patients $(19 \%)$ were considered ineligible for any anticoagulant for one of the following reasons: PLT $<30.000 /$ mmc $(n=20)$, active gastrointestinal bleeding $(n=2)$, moderate to severe renal failure $(n=1)$. Treatment discontinuation was necessary in $22(18.5 \%)$ patients: 11 for major bleeding episodes, 10 for a PLT count decreasing below $30.000 / \mathrm{mmc}$ and 1 for renal failure occurring after AT beginning. Forty-seven patients underwent a long-term 
Table 2 Characteristics of Antithrombotic Treatments for Eligible Cirrhotic Patients. Data are Expressed as Median (Interquartile Range) or Number (Percentage)

\begin{tabular}{|l|c|}
\hline AC treatment (n, \%) & $96(81 \%)$ \\
\hline LMWH treatment (n, \%) & $69(72 \%)$ \\
- therapeutic dose (n) & 40 \\
- prophylactic dose (n) & 29 \\
\hline VKA (n, \%) & $21(22 \%)$ \\
- INR 2-3 (n) & 14 \\
- INR I.8-2.5 (n) & 6 \\
- INR I.8-2.2 (n) & 1 \\
\hline Fondaparinux (n, \%) & $6(6 \%)$ \\
- therapeutic dose (n) & 5 \\
- prophylactic dose (n) & 1 \\
\hline
\end{tabular}

Abbreviations: LMWH, Low-molecular-weight heparin; VKA, Vitamin K antagonist.

AT treatment, defined as a (minimum of) six-month period of AT without any discontinuation. The median duration of AT was 8.5 months (range 1-15).

Among patients with incidentally detected cirrhotic SVT, 64 (77\%) underwent AT.

\section{Bleeding Events}

During follow-up, eleven MB events (10 upper digestive haemorrhages; 1 large muscle hematoma) occurred, corresponding to an incidence rate of 5.98 events per 100 patient years (95\% CI, 2.98-10.7). The incidence rate of MB was 8.4 events per 100 patient-years (95\% CI, 3.8315.97) in the subgroup of patients receiving AT therapy. According to treatment strategies, 8 bleedings occurred with intermediate-therapeutic doses of LWMH, 1 with prophylactic doses of LWMH and 2 with VKA. The incidence of major bleeding episodes among patients who suspended treatment was 4.76 per 100 patient-years $(95 \%$ CI: 0.53-17.19). In incidental SVT treated with AT, MB incidence was 6.5 per 100 patient-years (95\% CI: $2.8-$ 12.82) while in symptomatic SVT it was 2.2 per 100 patient-years (95\% CI: $0.25-8.02$ ).

\section{Thrombotic Events}

During AT treatment, 6 thrombotic events occurred (5 deep venous thrombosis (DVT) and 1 pulmonary embolism (PE), with an incidence of 5.6 per 100 patient/year (95\% CI, 2.05-12.2)). After discontinuation of AT, 4 thrombotic events occurred (2 DVT, 1 PE, 1 myocardial infarction), corresponding to an incidence of 9.52 per 100 patient/year (95\% CI, 2.56-24.38). The median time between discontinuation of AT and the vascular event was 10 months (range 2-24 months). All thrombotic events occurred in patients with incidental SVT, with an overall incidence of 7.75 per 100 patient/year $(95 \% \mathrm{CI}$, 3.71-14.26).

\section{Vessel Recanalization}

Seventy-eight $(81.2 \%)$ cirrhotic patients undergoing AT treatment had at least one follow-up imaging test, with the timing of the first test ranging from 1.3 to 3.7 months. The median number of imaging test per person was 2 (range 1-3). The radiological evaluation showed stability of SVT in 24 patients (31\%), extension/worsening in 6 $(7.5 \%)$, reduction in $14(18 \%)$ and total resolution in 34 (43.5\%). For cirrhotic patients in AT, the median time to complete recanalization was 6.58 months (IQR: 1.3-68.3).

Considering incidental cirrhotic SVT, imaging scans were available for 50 patients undergoing AT (78\%). The radiological evaluation showed stability of SVT in 13 patients $(26 \%)$, extension/worsening in $5(10 \%)$, reduction in $10(20 \%)$ and total resolution in $22(44 \%)$. Among the 23 cirrhotic patients not eligible for AT, 17 had an interim CT scan, which showed spontaneous improvement of SVT in $8(47 \%)$ and stability or worsening of SVT in $9(53 \%)$. In cirrhotic SVT, AT was not associated with higher recanalization rates $(\mathrm{p}=0.2)$. No differences, in terms of recanalization rates, were found comparing the different AT strategies (EBPM $(p=0.39)$; Fondaparinux $(p=0.48)$; AVK $(p=0.18)$ ). Moreover, the number of sites involved (single site vs multiple sites, $\mathrm{p}=0.27$ ) and the extension of SVT (total vs partial, $\mathrm{p}=0.36$ ) did not affect the recanalization rate.

Regarding the whole cirrhotic population on AT, the presence of HCC or solid cancer, was inversely associated with partial or total vessel recanalization (HR 0.46, 95\% CI $0.29-0.73, \mathrm{p}<0.01)$ at multivariate analysis.

\section{Functional and Survival Outcomes}

Thirty-two deaths occurred during the follow-up, with an incidence of 12.6 per 100 patients/year (95\% CI 8.6-17.8). Fourteen deaths were related to the underlying disease ( 8 for cancer progression, 6 for progressive liver failure); while in the remaining 18 cases the cause could not be ascertained. No bleeding- nor thrombotic-related deaths were recorded. Ten patients $(8.4 \%)$, underwent liver transplantation after vessel recanalization. Considering both treated and untreated cirrhotic patients, overall survival was significantly higher in 


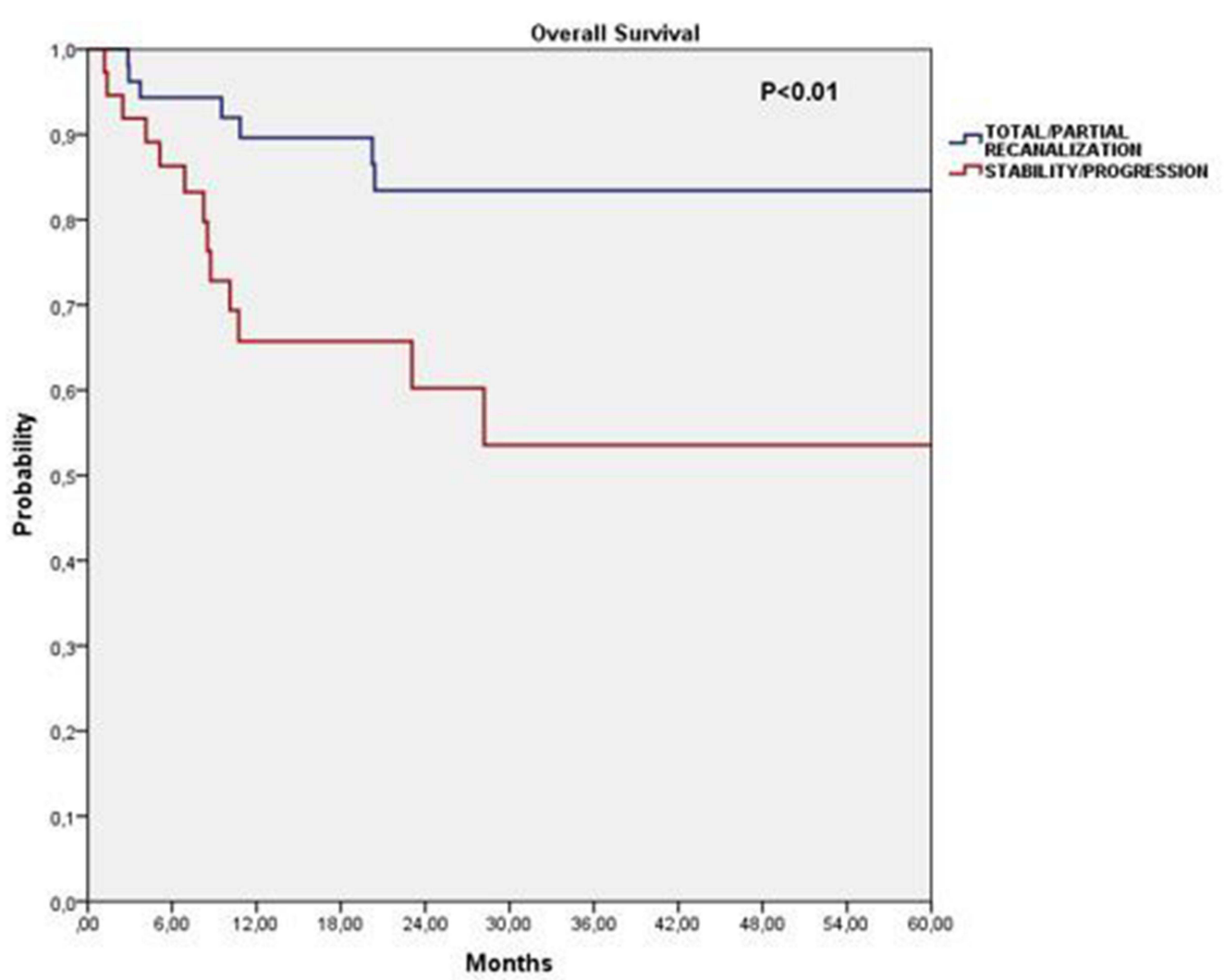

NUMBER AT RISK

$\begin{array}{llllllllllll}\text { TOTAL/PARTIAL RECANALIZATION } & 56 & 46 & 38 & 32 & 27 & 22 & 16 & 11 & 11 & 11 & 11 \\ \text { STABIUTY/PROGRESSION } & 39 & 31 & 18 & 13 & 11 & 8 & 6 & 6 & 4 & 3 & 3\end{array}$

Figure 3 Overall survival of cirrhotic patients, according to TVS recanalization.

patients who had at least a partial SVT recanalization at the first imaging test $(\mathrm{p}<0.01$, Figure 3$)$.

On multivariate analysis, the presence of $\mathrm{HCC}$ was an independent negative factor for overall survival (HR 0.48, 95\% CI 0.29-0.82, p < 0.01). AT was not associated with improved overall survival in cirrhotic patients $(p=0.2)$.

Regarding liver function, Child-Pugh score improved from baseline to last follow-up visit in 35 cirrhotic patients $(36.5 \%)$ on AT, while MELD score improved in 41 patients (43\%). At multivariate analysis, partial or total vessel recanalization resulted as an independent factor associated with MELD score reduction (HR 2.62, 95\% CI 1.1-6.47, $\mathrm{p}=0.03$ ) (Table 3), while no factor was associated with decreased Child-Pugh score.

\section{Discussion}

Our study was initially aimed to assess the efficacy and safety of anticoagulation in an unselected cohort of 149

Table 3 Univariate and Multivariate Cox Regression Analysis of the Factors Affecting MELD Score Improvement

\begin{tabular}{|c|c|c|c|c|c|c|}
\hline \multirow[t]{2}{*}{ Variable } & \multirow{2}{*}{$\begin{array}{c}\text { Univariate* } \\
\mathbf{p}\end{array}$} & \multirow[b]{2}{*}{ OR } & \multirow[b]{2}{*}{$95 \% \mathrm{Cl}$} & \multicolumn{3}{|c|}{ Multivariate } \\
\hline & & & & $\mathbf{p}$ & OR & $95 \% \mathrm{Cl}$ \\
\hline Age (>60 years old $v s<60$ years old) & 0.603 & 1.203 & $0.6-2.4$ & & & \\
\hline Ascites (yes vs no) & 0.881 & 1.056 & $0.5-2.2$ & & & \\
\hline Esophageal Varices (yes vs no) & 0.412 & 0.727 & $0.3-1.6$ & & & \\
\hline HCC or solid cancer (yes vs no) & 0.619 & 1.209 & $0.6-2.6$ & & & \\
\hline At least partial recanalization (yes vs no) & 0.043 & 2.720 & $\mathrm{I}-7.2$ & 0.03 & 2.62 & $\mathrm{I} . \mathrm{I}-6.47$ \\
\hline
\end{tabular}

Notes: *The factors with $\mathrm{P}<0.05$ in univariate analysis were enrolled into the forward stepwise multivariate Cox hazard regression.

Abbreviations: OR, Odds ratio; $\mathrm{Cl}$, confidence interval. 
patients with SVT. A pre-planned multidisciplinary treatment and follow-up algorithm was adopted in all cirrhotic SVT.

To date, few registries and no prospective studies have evaluated the impact of AT in cirrhotic SVT. One large cohort study, the International Registry on SVT (IRSVT), substantially supported the safety and efficacy of AT in most patients with SVT. ${ }^{3}$ Despite the clinical benefit attributed to AT, patients with SVT had a substantial long-term risk of thrombotic events (incidence rate 7.3 per 100 patient-years) that doubled the incidence rate of major bleedings (3.8 per 100 patient-years). As expected, the magnitude of risk was strongly influenced by the underlying clinical conditions, with a higher risk of both major bleeding and vascular events in liver cirrhosis than in other patient subgroups. A subsequent analysis of this same database on the subgroup with SVT associated with cirrhosis, confirmed the high risk of thrombotic events, which can be reduced by AT without increasing bleeding risk. ${ }^{14}$

LMWH and VKA are the cornerstones of AT for $\mathrm{SVT}^{7-10,15-17}$ and real-life data from the IRSVT showed that two-thirds of SVT patients switched from parenteral therapy to VKA, after a few days of overlapping treatment; in the subgroup of cirrhosis, $40 \%$ of patients continued with parenteral AT alone, with different doses and regimens. $^{14}$

The results of our study were comparable with the findings of Senzolo et $\mathrm{al}^{14}$ regarding the indication and type of AT administered (parenteral versus VKA). MB incidence per 100 patient-years was lower in our study, both during follow-up (6.0 and 9.8, respectively) and during AT (8.4 and 10.2 respectively) but slightly higher after AT discontinuation (4.7 and 3.1, respectively).

In 2012, 12 years after the first enrolment of our study, the 9th edition of the American College of Chest Physician (ACCP) guidelines ${ }^{9}$ strongly recommended anticoagulant therapy in symptomatic patients. Conversely, no treatment was suggested for incidentally detected SVT. Recently, results of two large cohort studies suggested that the prognosis of incidentally detected SVT is comparable to symptomatic SVT. In the study by Riva et al ${ }^{18}$ an overall incidence rate of thrombotic events of 8.0 and 7.0 per 100 person-year was reported in incidentally detected and symptomatic SVT, respectively. In particular, untreated incidental SVT had an incidence rate of 11.5 events. Similar results came from the "Registro Informatizado Enfermedad Trombo Embólica" (RIETE) registry where patients with incidentally detected SVT presented a non-significantly higher rate of symptomatic recurrent VTE than those with symptomatic SVT. ${ }^{19}$ Being aware of the persisting limitations of therapy with LMWH/ fondaparinux (compliance issues, inaccessibility for follow-up and renal failure) and VKA (spontaneous INR prolongation and interference with MELD score), we considered all patients eligible for AT anyway. Taking into account the evidence of hypercoagulability and rebalancing of haemostasis in liver cirrhosis ${ }^{20,21}$ and questioning the role of the "wait and see" policy in the management of SVT, we promoted an early use of anticoagulants (both parenteral and VKA) in the absence of absolute contraindications, to reduce the rate of thrombotic recurrences, achieve high rate of recanalization and delay occurrence of hepatic decompensation. In this regard, the incidence of thrombotic events per 100 patient-years was lower in our study in comparison with Senzolo data, ${ }^{14}$ both during the whole study period (5.98 versus 12.9) and during AT (5.6 versus 9.1), but higher after AT discontinuation (9.52 versus 3.1, respectively). Notably, in our series, all thrombotic events occurred in patients with incidental SVT, with an overall incidence of 7.75 per 100 patient/year.

Regarding radiological follow-up, in our study, the choice of abdominal imaging was left to the attending physicians. Seventy-eight cirrhotic patients in AT underwent a CT scan that showed partial or total recanalization in $61.5 \%$ of the cases, a response rate similar to previous published studies. $^{3,22,23}$ The recanalization rate was similar $(64 \%)$ in the subgroup of patients with incidental SVT. In our series, vessel patency was at least partially restored in $47 \%$ of cirrhotic patients not eligible for AT, confirming previous results on spontaneous recanalization of SVT. $^{24,25}$

So far, conflicting data about the impact of SVT on the natural history of cirrhosis exist. Recent findings suggest that anticoagulation may interfere with liver fibrogenesis and consequently may be a potent tool for treating portal hypertension. ${ }^{26}$ Furthermore, a recent meta-analysis found that early AT significantly increased portal vein recanalization, did not significantly influence bleeding rates and it was associated with increased overall survival. ${ }^{27}$ Longterm effectiveness and safety of LMWH as a prophylaxis strategy in advanced cirrhotic patients was demonstrated by Villa et al in a small randomized controlled trial, in which a 12-month course of enoxaparin prevented portal vein thrombosis and appeared to delay the occurrence of hepatic decompensation and to improve survival. ${ }^{28}$ A recent paper from Nery et al suggested that untreated 
PVT may undergo spontaneous recanalization without significantly worsening the course of cirrhosis. ${ }^{23}$ Conversely, in the paper from Luca et $\mathrm{al}^{25}$ and Senzolo et $\mathrm{al}^{14}$ a trend to higher mortality rates was found in patients with no radiological evidence of vessel recanalization, especially in patients with more advanced liver disease. Our study confirmed these findings, as vessel recanalization was associated with a higher overall survival and a MELD score improvement, suggesting, like Senzolo et $\mathrm{al}^{14}$ that restored vessel patency determines a functional improvement in severe cirrhotic patients.

In addition, recent data suggested that the amelioration of liver dysfunction explored by MELD score may predict the improvement of SVT in liver cirrhosis. ${ }^{29}$

The best way to obtain a vessel recanalization (eg, no treatment, EBPM, Fondaparinux, VKA, NOACs) is still under debate and unequivocal conclusions from our study cannot be drawn, since no treatment strategy showed an increased recanalization rate compared to the others.

The present study has several limitations due to its observational design. As a result of the complex scenario of SVT, the possibility that initial selection bias may have occurred at the time of patient enrolment cannot be excluded, but we believe that the multidisciplinary longterm surveillance was accurate as well as the completeness of the routinely collected data. Clearly, in addition to anticoagulation, other therapeutic strategies like oesophageal varices prophylaxis (beta-blockers and/or endoscopic ligatures), Transjugular Intrahepatic Porto-systemic Shunt and Orthotopic Liver Transplant largely influenced SVT outcome.

Another important limitation is the fact that follow-up SVT imaging was not done according to a strict protocol at fixed times. However, all the CT scans were obtained within 4 months from the diagnosis of SVT, so that we could analyze any association between radiological findings and functional outcome and/or overall survival. The variable timing of imaging during the follow-up prevented us from drawing any conclusions regarding the time to recanalization.

In conclusion, despite potential pitfalls and caveats, our study confirms that, in cirrhotic SVT patients, partial or total resolution of thrombosis improves liver function and reduces mortality. Considering our actual practice with the existing evidence, we believe that combining the accurate identification and the correction of the baseline bleeding risk whenever it is possible, together with a stringent multi-disciplinary monitoring of both thrombotic and haemorrhagic events and dynamic recanalization studies at fixed times, we could further optimize SVT management in the future. Moreover, preliminary data on DOACs use in cirrhotic $\mathrm{SVT}^{30-36}$ suggest that the time has come to perform randomized quality studies to compare traditional with newer anticoagulants.

\section{Abbreviations}

AT, Anticoagulation Therapy; SVT, Splanchnic Vein Thrombosis; DVT, Deep Venous Thrombosis; PE, Pulmonary Embolism; HCC, HepatoCellular Carcinoma; GFR, Glomerular Filtration Rate; LMWH, Low Molecular Weight Heparin; VKA, Vitamin K Antagonists; MB, Major Bleeding; PE, Pulmonary Embolism; VTE, Venous Thromboembolism; DOAC, Direct-Acting Oral Anticoagulants; EGD, Esophagogastroduodenoscopy.

\section{Acknowledgments}

The authors would like to thank the patients, their parents and all the nurses and physicians at the Center who all contributed to the management of patient care.

\section{Authorship Contributions}

All authors made a significant contribution to the work reported, whether that is in the conception, study design, execution, acquisition of data, analysis and interpretation, or in all these areas; took part in drafting, revising or critically reviewing the article; gave final approval of the version to be published; have agreed on the journal to which the article has been submitted; and agree to be accountable for all aspects of the work.

\section{Disclosure}

No conflict of interest for any of the authors. The authors did not receive any financial support. All the authors designed the study, contributed patients, helped abstract patient information, performed statistical analysis, and wrote the paper.

\section{References}

1. Myers K, Hannah P. Anatomy of veins and lymphatics. In: Myers K, Hannah P, editors. Manual of Venous and Lymphatic Diseases. London, UK: Taylor \& Francis Ltd; 2018:13-38.

2. Thatipelli MR, McBane RD, Hodge DO, et al. Survival and recurrence in patients with splanchnic vein thromboses. Clin Gastroenterol Hepatol. 2010;8(2):200-205. doi:10.1016/j.cgh.2009.09.019

3. Ageno W, Riva N, Schulman S, et al. Long-term clinical outcomes of splanchnic vein thrombosis: results of an international registry. JAMA Intern Med. 2015;175(9):1474-1480. doi:10.1001/jamainternm ed.2015.3184 
4. Ageno W, Squizzato A, Togna A, et al. Incidental diagnosis of a deep vein thrombosis in consecutive patients undergoing a computed tomography scan of the abdomen: a retrospective cohort study. $J$ Thromb Haemost. 2012;10(1):158-160. doi:10.1111/j.15387836.2011.04565.x

5. Ageno W, Dentali F, Squizzato A. How I treat splanchnic vein thrombosis. Blood. 2014;124(25):3685-3691. doi:10.1182/blood2014-07-551515

6. Valeriani E, Riva N, Di Nisio M, et al. Splanchnic vein thrombosis: current perspectives. Vasc Health Risk Manag. 2019;15:449-461. doi:10.2147/VHRM.S197732

7. European Association for the Study of the Liver. EASL clinical practice guidelines: vascular diseases of the liver. $J$ Hepatol. 2016;64(1):179-202. doi:10.1016/j.jhep.2015.07.040

8. De Franchis R, Faculty BV. Expanding consensus in portal hypertension: report of the Baveno VI Consensus Workshop: stratifying risk and individualizing care for portal hypertension. J Hepatol. 2015;63 (3):743-752. doi:10.1016/j.jhep.2015.05.022

9. Kearon C, Akl EA, Comerota AJ, et al. Antithrombotic therapy for VTE disease: antithrombotic therapy and prevention of thrombosis, 9th ed: American College of Chest Physicians evidence-based clinical practice guidelines. Chest. 2012;141(2 Suppl):e419S-e496S.

10. DeLeve LD, Valla DC, Garcia-Tsao G, et al. Vascular disorders of the liver. Hepatology. 2009;49(5):1729-1764.

11. Basili S, Pastori D, Raparelli V, et al. Anticoagulant therapy in patients with liver cirrhosis and portal vein thrombosis: insights for the clinician. Therap Adv Gastroenterol. 2018;6(11):1-10.

12. Flores B, Trivedi HD, Robson SC, et al. Hemostasis, bleeding and thrombosis in liver disease. J Transl Sci. 2017;3(3). doi:10.15761/ JTS.1000182.

13. Schulman S, Kearon C. Subcommittee on Control of Anticoagulation of the Scientific and Standardization Committee of the International Society on Thrombosis and Haemostasis. Definition of major bleeding in clinical investigations of antihemostatic medicinal products in non-surgical patients. J Thromb Haemost. 2005;3(4):692-694.

14. Senzolo M, Riva N, Dentali F, et al. Long-term outcome of splanchnic vein thrombosis in cirrhosis. Clin Transl Gastroenterol. 2018;9 (8):176. doi:10.1038/s41424-018-0043-2

15. Riva N, Ageno W. Approach to thrombosis at unusual sites: splanchnic and cerebral vein thrombosis. Vasc Med. 2017;22(6):529-540. doi:10.1177/1358863X17734057

16. Senzolo M, Riggio O, Primignani M. Vascular disorders of the liver: recommendations from the Italian Association for the Study of the Liver (AISF) ad hoc committee. Dig Liver Dis. 2011;43(7):503-514. doi:10.1016/j.dld.2010.11.006

17. Riva N, Ageno W, Poli D, et al. Safety of vitamin K antagonist treatment for splanchnic vein thrombosis: a multicenter cohort study. J Thromb Haemost. 2015;13(6):1019-1027. doi:10.1111/ jth. 12930

18. Riva N, Ageno W, Schulman S, et al. Clinical history and antithrombotic treatment of incidentally detected splanchnic vein thrombosis: a multicentre, international prospective registry. Lancet Haematol. 2016;3(6):e267-e275. doi:10.1016/S2352-3026(16)30020-5

19. Tufano A, Ageno W, Di Micco P, et al. Outcomes during anticoagulation in patients with symptomatic vs. incidental splanchnic vein thrombosis. Thromb Res. 2018;164:69-74. doi:10.1016/j. thromres.2018.02.143

20. Tripodi A, Primignani M, Chantarangkul V, et al. An imbalance of pro- vs anti-coagulation factors in plasma from patients with cirrhosis. Gastroenterology. 2009;137(6):2105-2111. doi:10.1053/j. gastro.2009.08.045
21. Lisman T, Porte RJ. Rebalanced hemostasis in patients with liver disease: evidence and clinical consequences. Blood. 2010;116 (6):878-885. doi:10.1182/blood-2010-02-261891

22. Werner KT, Sando S, Carey EJ, et al. Portal vein thrombosis in patients with end stage liver disease awaiting liver transplantation: outcome of anticoagulation. Dig Dis Sci. 2013;58:1776-1780. doi:10.1007/s10620-012-2548-y

23. Nery F, Chevret S, Condat B, et al. Causes and consequences of portal vein thrombosis in 1,243 patients with cirrhosis: results of a longitudinal study. Hepatology. 2015;61(2):660-667. doi:10.1002/ hep. 27546

24. Qi X, Guo X, Yoshida EM, et al. Transient portal vein thrombosis in liver cirrhosis. BMC Med. 2018;16(1):83. doi:10.1186/s12916-0181069-8

25. Luca A, Caruso S, Milazzo M, et al. Natural course of extrahepatic nonmalignant partial portal vein thrombosis in patients with cirrhosis. Radiology. 2012;265(1):124-132. doi:10.1148/radiol.12112236

26. Turco L, Schepis F, Villa E. The role of anticoagulation in treating portal hypertension. Current Hepatol Rep. 2018;17:200-208.

27. Le W, Guo X, Xu X, et al. Anticoagulation favors thrombus recanalization and survival in patients with liver cirrhosis and portal vein thrombosis: results of a meta analysis. Adv Ther. 2020;38 (1):495-520.

28. Villa E, Cammà C, Marietta M, et al. Enoxaparin prevents portal vein thrombosis and liver decompensation in patients with advanced cirrhosis. Gastroenterology. 2012;143(5):1253-1260.e4. doi:10.10 53/j.gastro.2012.07.018

29. Xu S, Guo X, Xu X, et al. Natural history and predictors associated with the evolution of portal venous system thrombosis in liver cirrhosis. Eur J Gastroenterol Hepatol. 2021. doi:10.1097/ MEG.0000000000002123

30. Hanafy AS, Abd-Elsalam S, Dawoud MM. Randomized controlled trial of rivaroxaban versus warfarin in the management of acute non-neoplastic portal vein thrombosis. Vascul Pharmacol. 2019;113:86-91.

31. Nagaoki Y, Aikata H, Daijyo K, et al. Efficacy and safety of edoxaban for treatment of portal vein thrombosis following danaparoid sodium in patients with liver cirrhosis. Hepatol Res. 2018;48 (1):51-58. doi:10.1111/hepr.12895

32. Janczak DT, Mimier MK, McBane RD, et al. Rivaroxaban and apixaban for initial treatment of acute venous thromboembolism of atypical location. Mayo Clin Proc. 2018;93(1):40-47. doi:10.1016/j. mayocp.2017.10.007

33. Naymagon L, Tremblay D, Zubizarreta N, et al. The efficacy and safety of direct oral anticoagulants in noncirrhotic portal vein thrombosis. Blood Adv. 2020;4(4):655-666. doi:10.1182/ bloodadvances. 2019001310

34. Naymagon L, Tremblay D, Zubizarreta N, Moshier E, Mascarenhas J, Schiano T. Safety, efficacy, and long-term outcomes of anticoagulation in cirrhotic portal vein thrombosis. Dig Dis Sci. 2020. doi:10.1007/s10620-020-06695-4

35. Intagliata NM, Henry $\mathrm{ZH}$, Maitland $\mathrm{H}$, et al. Direct oral anticoagulants in cirrhosis patients pose similar risks of bleeding when compared to traditional anticoagulation. Dig Dis Sci. 2016;61:1721-1727. doi:10.1007/s10620-015-4012-2

36. Hanafy AS, Abd-Elsalam S, Dawoud MM. Randomized controlled trial of rivaroxaban versus warfarin in the management of acute non-neoplastic portal vein thrombosis. Vascul Pharmacol. 2019;113:86-91. 


\section{Publish your work in this journal}

Vascular Health and Risk Management is an international, peerreviewed journal of therapeutics and risk management, focusing on concise rapid reporting of clinical studies on the processes involved in the maintenance of vascular health; the monitoring, prevention and treatment of vascular disease and its sequelae; and the involvemen of metabolic disorders, particularly diabetes. This journal is indexed on PubMed Central and MedLine. The manuscript management system is completely online and includes a very quick and fair peerreview system, which is all easy to use. Visit http://www.dovepress. com/testimonials.php to read real quotes from published authors. 